\title{
A modern multidisciplinary approach to a large cervicothoracic chordoma using staged en bloc resection with intraoperative image-guided navigation and 3D-printed modeling: illustrative case
}

\author{
*Nathan J. Pertsch, BA, ${ }^{1}$ Owen P. Leary, ScB, $, 1,2$ Joaquin Q. Camara-Quintana, MD, ${ }^{1,2}$ David D. Liu, ScB, ${ }^{1}$ Tianyi Niu, MD, $, 1,2$ \\ Albert S. Woo, MD, ${ }^{1,3}$ Thomas T. Ng, MD, ${ }^{1,4}$ Adetokunbo A. Oyelese, MD, PhD, ${ }^{1,2}$ Jared S. Fridley, MD, ${ }^{1,2}$ and Ziya L. Gokaslan, MD ${ }^{1,2}$ \\ ${ }^{1}$ The Warren Alpert Medical School of Brown University, Providence, Rhode Island; and Departments of ${ }^{2}$ Neurosurgery, ${ }^{3}$ Plastic Surgery, and ${ }^{4}$ Thoracic Surgery, \\ Rhode Island Hospital, Providence, Rhode Island
}

\begin{abstract}
BACKGROUND Cervicothoracic junction chordomas are uncommon primary spinal tumors optimally treated with en bloc resection. Although en bloc resection is the gold standard for treatment of mobile spinal chordoma, tumor location, size, and extent of involvement frequently complicate the achievement of negative margins. In particular, chordoma involving the thoracic region can require a challenging anterior access, and en bloc resection can lead to a highly destabilized spine.

OBSERVATIONS Modern technological advances make en bloc resection more technically feasible than ever before. In this case, the successful en bloc resection of a particularly complex cervicothoracic junction chordoma was facilitated by a multidisciplinary surgical approach that maximized the use of intraoperative computed tomography-guided spinal navigation and patient-specific three-dimensional-printed modeling.

LESSONS The authors review the surgical planning and specific techniques that facilitated the successful en bloc resection of this right-sided chordoma via image-guided parasagittal osteotomy across 2 stages. The integration of emerging visualization technologies into complex spinal column tumor management may help to provide optimal oncological care for patients with challenging primary tumors of the mobile spine.
\end{abstract}

https://thejns.org/doi/abs/10.3171/CASE2023

KEYWORDS spinal oncology; cervicothoracic junction; 3D printing; en bloc resection; multidisciplinary surgical teams

Chordomas are uncommon primary spinal column tumors arising from embryonic remnants of the notochord. They have a male predominance, a peak incidence in the 5 th and 6th decades of life, ${ }^{1-4}$ and an estimated overall incidence of 0.18 to 0.8 per $1,000,000$ persons per year. ${ }^{5}$ Median survival is estimated at 6.29 years. ${ }^{1}$ The majority of chordomas arise in the sacrococcygeal (45-50\%) and sphenooccipital (35-40\%) regions, with only 10-15\% occurring in the mobile (C1-L5) spine. ${ }^{1}$ Typically, chordoma is slow growing and invasive; in the mobile spine, recurrence is seen in $60-100 \%$ of cases without en bloc resection. ${ }^{1,6-8}$

Definitive management of chordoma is en bloc resection with wide margins to preserve the tumor capsule, minimize residual tumor cells, and reduce the risk of recurrence..$^{2,3,7-10}$ En bloc resection is technically demanding, but substantial literature supports its use and efficacy in the mobile spine. , $^{11-18}$ These lesions often require a challenging anterior access through the thoracic or peritoneal cavities and can lead to significant spinal destabilization requiring innovative approaches to reconstruction, stabilization, and fusion. Adaptations to improve the feasibility of en bloc resection have included multistaged surgeries, collaboration across surgical specialties, minimally invasive techniques, and image-guided navigation. ${ }^{11,12,15,17,19-21}$

Through this case, we describe the multidisciplinary surgical management of a large cervicothoracic chordoma in which intraoperative utilization of computed tomography (CT)-guided navigation and patientspecific three-dimensional (3D)-printed tumor modeling aided a successful 2-stage en bloc tumor resection and spinal reconstruction.

ABBREVIATIONS 3D = three-dimensional; $\mathrm{CT}=$ computed tomography; $\mathrm{CTA}=$ computed tomography angiography; $\mathrm{MRI}=$ magnetic resonance imaging. INCLUDE WHEN CITING Published February 8, 2021; DOI: 10.3171/CASE2023.

SUBMITTED October 20, 2020. ACCEPTED November 11, 2020.

* N.J.P. and O.P.L. contributed equally to this work.

(c) 2021 The authors, CC BY-NC-ND 4.0 (http://creativecommons.org/licenses/by-nc-nd/4.0/). 


\section{Illustrative Case}

A 68-year-old male with no significant medical history presented to the outpatient clinic with 6 months of numbness, paresthesias, and progressive, severe, radicular pain along the medial aspect of his right upper extremity. He denied any left-sided pain, lower extremity weakness, balance problems, and bowel or bladder symptoms. On examination, decreased pinprick sensation was observed in the right C8 and T1 nerve distributions with full preservation of strength. He subsequently developed a right-sided miosis, ptosis, and anhidrosis characteristic of Horner syndrome.

\section{Diagnosis and Staging}

Magnetic resonance imaging (MRI) revealed an intrathoracic tumor occupying the apex of the right lung with significant right-to-left deviation of both the trachea and esophagus. The lesion appeared to involve the right-sided 1st-4th ribs, C6-T4 vertebral bodies, C8-T4 neural foramina, brachial plexus, brachiocephalic trunk, subclavian artery, and vertebral artery, without intraspinal epidural extension (Fig. 1). The tumor measured $9.3 \mathrm{~cm}$ (craniocaudal) by $6.2 \mathrm{~cm}$ (leftright) by $6.8 \mathrm{~cm}$ (anterior-posterior). A Pancoast tumor was initially suspected, but positron emission tomography imaging demonstrated minimal fluorodeoxyglucose uptake, and CT-guided biopsy revealed physaliferous cells with positive brachyury staining, both consistent with chordoma. The tumor was Enneking stage IB.

\section{Preoperative Planning}

Due to invasion of spinal and intrathoracic structures, en bloc resection necessitated combined anterior and posterior approaches. First, an anterior approach would be used to free the tumor from thoracic, neurovascular, and osseous structures and to perform multilevel parasagittal vertebral body osteotomies. Second, a posterior approach would permit en bloc resection of the tumor, along with involved posterior vertebral elements, chest wall, and T1-T4 nerve roots.

Additional preoperative imaging was obtained, including highresolution MRI of the spine, brain, and right shoulder (T1- and T2weighted sequences) and CT angiography (CTA) of the neck. These studies facilitated tumor mapping for intraoperative stereotactic navigation and creation of a precise, full-size, 3D-printed model of the tumor and adjacent anatomy (Fig. 1). Both the 3D-printed model and the CTA demonstrated that the tumor completely encased the right vertebral artery, indicating intraoperative sacrifice to achieve en bloc resection. The CTA indicated that bilateral vertebral arteries were codominant.

\section{Stage I: Anterior Trap-Door Approach to the Cervicothoracic Junction With Parasagittal Vertebral Body Osteotomy}

Thoracic surgery personnel performed an anterolateral cervical incision and right-sided trap-door thoracotomy ${ }^{22}$ extending through
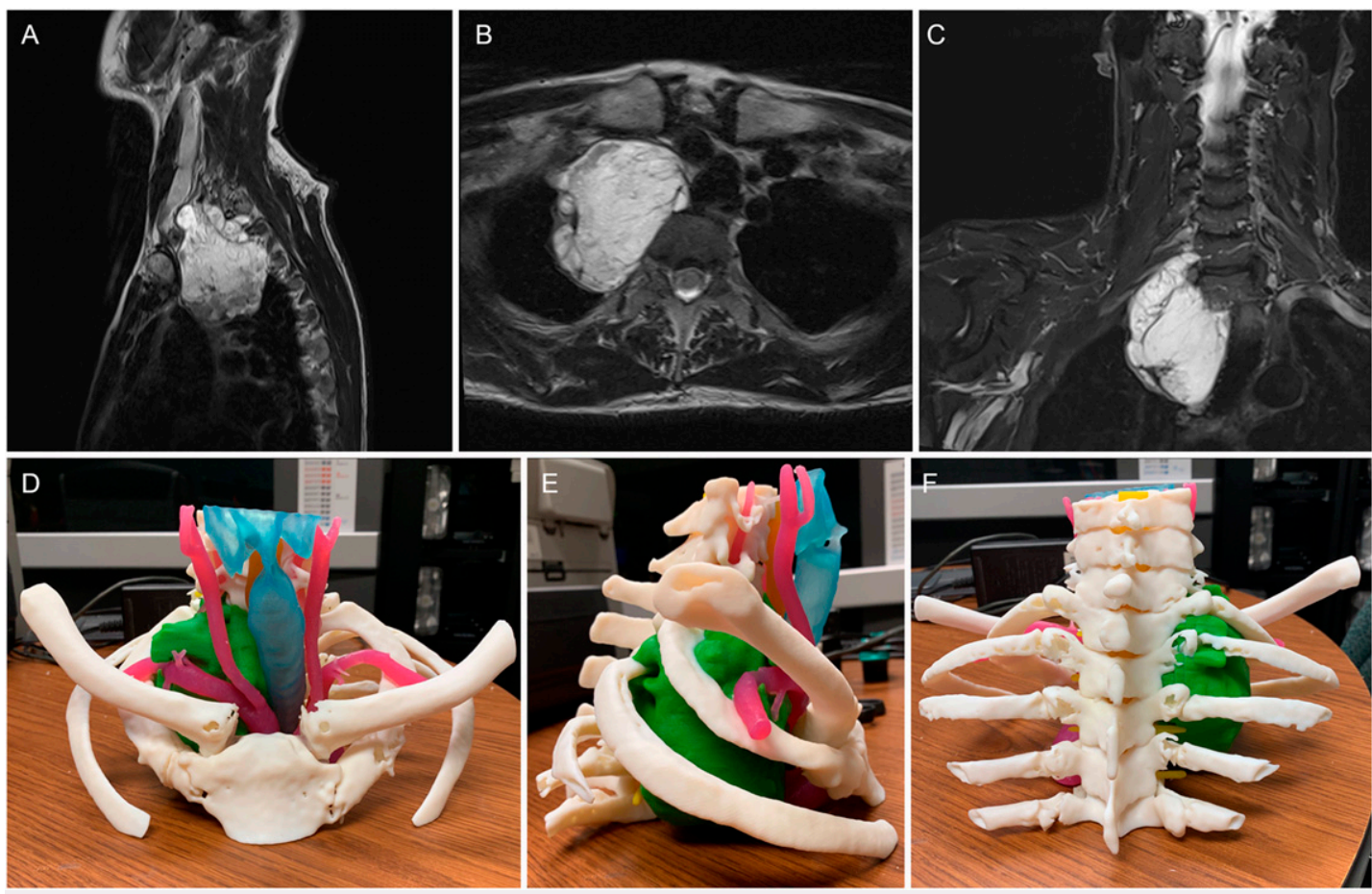

FIG. 1. T2-weighted MR images of the cervicothoracic chordoma in sagittal (A), axial (B), and coronal (C) planes of view, demonstrating extensive involvement of adjacent chest wall and vascular structures, as well as right-to-left tracheal and esophageal displacement. A full-size plastic 3D model (D-F) was printed using layered manufacturing on a Stratasys J750 printer (StrataSys Ltd.) after virtual reconstruction of the patient's anatomy from preoperative MRI and CTA imaging sets. Anatomies of interest were manually or semi-automatically segmented using 3D Slicer (v. 4.10, Massachusetts Institute of Technology and Brigham and Women's Hospital), including bone (white), trachea (blue), and arterial blood flow (pink) from the CTA, as well as tumor (green), esophagus (brown), intervertebral discs (blue), and spinal cord and nerve roots (yellow) from the T2-weighted MRI. 
the 4th intercostal space and involving bisection of the sternum to expose the vertebral bodies of the cervicothoracic junction anteriorly (Fig. 2). Anterior structures were mobilized and separated from the tumor capsule. The right-sided vertebral artery was isolated as much as possible but could not be completely skeletonized anteriorly.

Intraoperative spinal navigation and tumor mapping were set up using preoperative MRI and CT imaging and were utilized intraoperatively to localize critical neurovascular structures and bony anatomy relative to the tumor (Brainlab). The navigation reference array was clamped to the exposed 5th rib because of the absence of other adequate bony reference points following thoracotomy, and an intraoperative CT was obtained using an AIRO scanner (Brainlab) to register the image-guided navigation with patient anatomy.

Anterior parasagittal osteotomies were performed medial to the right-sided tumor through the C6-T4 vertebral bodies using a navigated drill equipped with a high-speed diamond burr. The right-sided 1st rib distal to the tumor mass was resected to detach the tumor anteriorly. A Silastic sheath was inserted between the tumor mass and the mobilized vessels, trachea, and esophagus to protect these structures during stage II. The sternum was reapproximated using steel wire and a chest tube was placed.

Stereotactic guidance was especially useful during the osteotomies to ensure a wide, medial margin through the vertebral bodies, which were partially invaded by the tumor more laterally. The 3D-printed model was reviewed extensively as a physical reference for the spatial relationships between key anatomical structures and assisted in realtime decision-making about the extent and sequence of mobilizing adjacent structures during the surgical approach.

After stage I, the patient was transferred to the neurocritical care unit and remained intubated overnight without complications in preparation for stage II the following day.

\section{Stage II: Posterior Approach to the Cervicothoracic Junction With En Bloc Resection of the Chordoma and Subsequent Posterior Cervicothoracic Instrumented Fusion}

We performed a posterior midline approach to the cervicothoracic spine, with right-sided $\mathrm{C} 6-\mathrm{T} 4$ hemilaminectomies to complete rightsided partial spondylectomies from C6 to T4. Posterior neurovascular elements were mobilized from the tumor; however, the right-sided T1-T4 nerve roots and right vertebral artery were ligated and cut due to suspected or visualized tumor involvement. The tumor was adherent to the upper lobe of the right lung and chest wall, requiring wedge resection by thoracic surgery of the involved lung and chest wall, including associated portion of the $1 \mathrm{st}-5$ th ribs. Once entirely detached, the tumor mass was removed en bloc (Fig. 2). Posterior cervicothoracic instrumented fusion with bilateral dual rods and cross connectors was performed from C2 to T9 using DePuy Mountaineer and Expedium systems for the cervical and thoracic portions of the construct, respectively. No right-sided screws were placed from C6 to T4 due to the hemicorpectomy defect.

AIRO intraoperative CT was used for the placement of thoracic pedicle screws and tumor mapping during stage II, with a reference array secured to the T9 spinous process. The 3D-printed model was used throughout the procedure, particularly during completion of partial spondylectomies. Upper- and lower-extremity motor evoked and somatosensory evoked potentials were used continuously throughout both stages, with no noted reduction in evoked potentials. Plastic surgery personnel closed the posterior defect by mobilizing and reapproximating paraspinous musculocutaneous flaps to minimize the risk of postoperative wound complications. ${ }^{23}$

\section{Pathology}

Surgical pathology confirmed a $9.0 \times 5.5 \times 8.0$-cm tumor with an intact tumor capsule and negative margins and noted invasion of tumor cells into the 1st rib and parietal pleura but not the wall of the resected right vertebral artery. Additionally, the tumor was found to invade the T1 and $T 2$ vertebral bodies, the corresponding intervertebral disc, and the C7 dorsal root ganglion.

\section{Postoperative Course}

The patient's immediate postoperative course was complicated by ileus, hypotension, new-onset atrial flutter, a decrease in right-sided grip strength to $3 / 5$ (which improved to $4 / 5$ ), and new left-sided vision loss attributed to underlying ischemic optic neuropathy. After 17 days, the patient was discharged to acute inpatient rehabilitation but was then readmitted on postoperative day 24 with right pleural effusion, pulmonary edema, and an anterior chest wall infection requiring surgical debridement. Blood and tissue cultures were positive for methicillinresistant Staphylococcus aureus, which was treated with 2 weeks of intravenous vancomycin. The patient was discharged home on postoperative day 42.

At the patient's 3-month outpatient follow-up, atrial flutter had resolved, but right-sided hand weakness related to planned sectioning of the T1 nerve root was still present at strength $4 / 5$. Improvement in pain was reported. Spinal imaging at the 4-month follow-up demonstrated the hardware to be well positioned without signs of failure (Fig. 3). At the 6-month follow-up, the patient complained of continued right-hand weakness as well as right shoulder and extremity neuropathic pain. Follow-up proton beam radiation to the surgical site was recommended per standard of care, but the patient declined, citing concern for plexopathy. At the 1-year follow-up, imaging continued to demonstrate a stable construct but with possible signs of residual or recurring disease on MRI; proton beam radiation was again recommended.

\section{Discussion \\ Observations}

In this case, a multidisciplinary team of surgeons successfully resected a particularly large cervicothoracic junction chordoma en bloc utilizing multiple increasingly common innovations in visualization technology to maximize precision and aid team communication. This case highlights the value of CT-guided navigation coupled with tumor mapping, which was particularly helpful given the tumor size and the involvement of several critical neurovascular structures.

Furthermore, preoperative high-resolution MRI and neck CTA enabled the creation of a 3D-printed model of the chordoma with surrounding bony, neurovascular, and visceral anatomy. Surgeons reported that the 3D-printed model improved spatial orientation and evaluation of local tumor involvement. The tactility of the model was especially helpful during intraoperative collaboration between thoracic and neurosurgery personnel, allowing for an enhanced appreciation of tumor proximity to vital structures and moment-to-moment operative planning.

\section{Lessons}

Multidisciplinary approaches and new technology are becoming increasingly common in treating mobile spine chordoma. ${ }^{13,24-28}$ These include strengthening interdisciplinary collaborations across neurosurgery, general surgery, thoracic surgery, head and neck surgery, plastic surgery, medical and radiation oncology, and rehabilitation services. This study reinforces the utility of a physical reference during multidisciplinary operations. Here, the 3D-printed model facilitated 

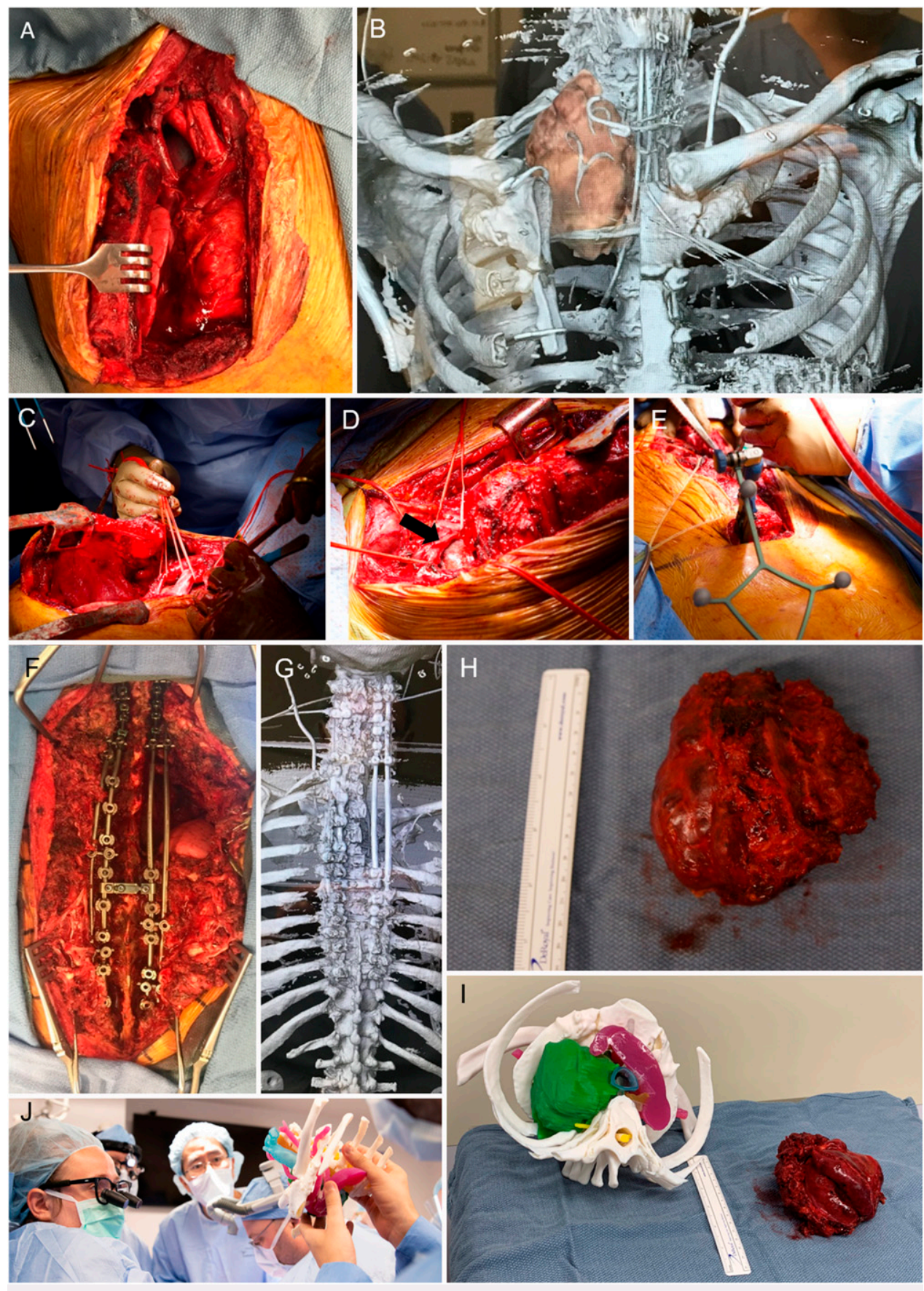

FIG. 2. An incision was planned from the neck incorporating the sternum and 4th intercostal space, and trapdoor thoracotomy with sternal bisection was performed to achieve anterior exposure of the spine $(\mathbf{A})$. An intraoperative CT scan was obtained and reviewed with tumor mapping to guide resection (B). Through the anterior access, adjacent vessels were retracted $(\mathbf{C})$, and the underlying tumor capsule was visible (D, arrow). A navigation array was secured to the right-sided 5 th rib $(\mathbf{E})$. After anterior mobilization of the tumor, including parasagittal osteotomy through the vertebral bodies, a second stage was performed from posteriorly. The contralateral (left-sided) rod was placed first, followed by the rest of the instrumentation consisting of double rods bilaterally as well as cross connectors $(\mathbf{F})$. Another intraoperative CT was performed to visualize the complete instrumentation after placement $(\mathbf{G})$. The defect in the chest wall with underlying structures was visible after tumor resection posteriorly ( $\mathbf{F}$ and $\mathbf{G})$. The tumor mass was resected with a visibly intact capsule $(\mathbf{H})$ and was compared side by side with the 3D model $(\mathbf{I})$. The model was used next to the operating table throughout both stages of the procedure, where it provided a convenient reference for key $3 \mathrm{D}$ relationships between structures, particularly when planning approach and osteotomy (J). 

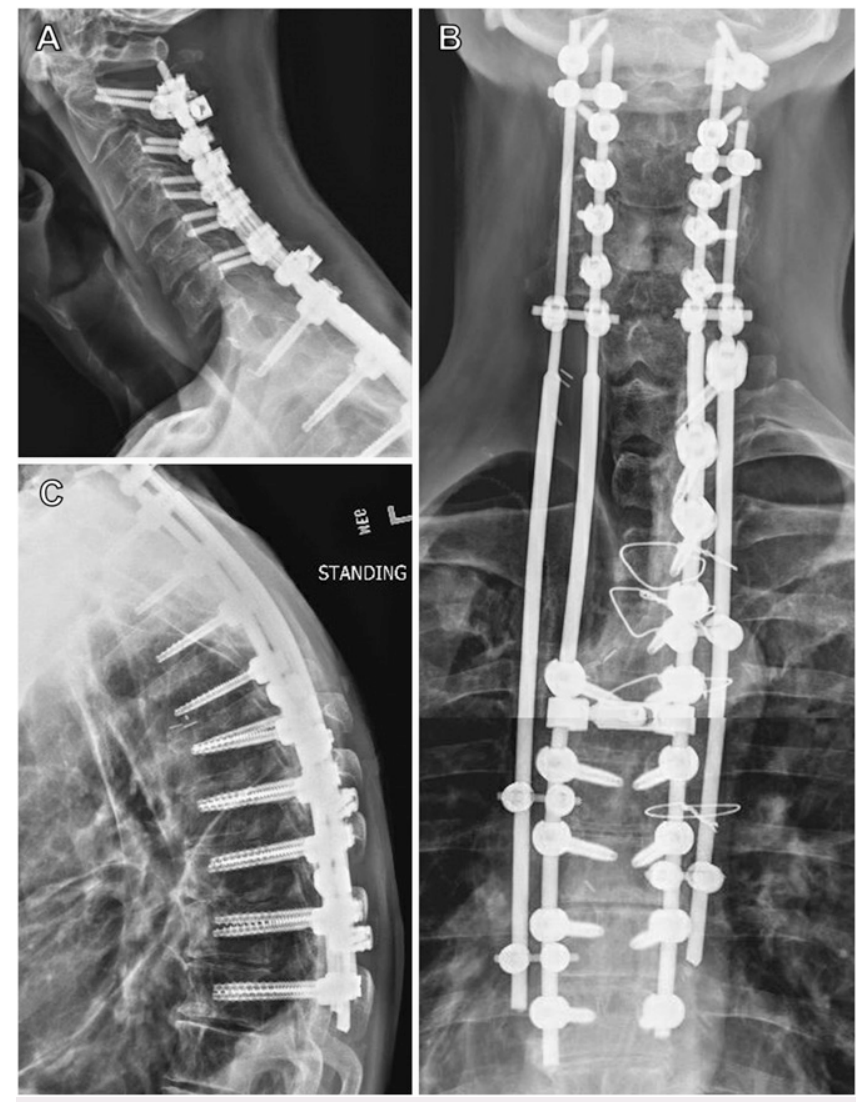

FIG. 3. Lateral (A and C) and anterior-posterior (B) radiographs taken 4 months postoperatively demonstrate well-positioned hardware without signs of construct failure. Steel wire used for sternal reapproximation is also visible in the anterior view.

communication of the operative plan between members of the neurological, plastic, and thoracic surgery teams. Furthermore, 3D-printed patient-specific modeling can be helpful for making procedural decisions. Here, it was useful for determining the best approach relative to neurovascular structures adjacent to, abutting, or invaded by tumor and for anticipating precise osteotomy planes when mobilizing the tumor from the vertebral column. Due to these and other benefits, the use of $3 \mathrm{D}$ printing for surgical planning in spinal surgery is increasing rapidly. ${ }^{29}$ However, within spinal oncology, reports have been limited to small case series and case studies. ${ }^{24,30-33}$ Larger-scale studies are needed to fully understand the benefits of this technology in spinal oncological surgery.

The use of intraoperative CT and stereotactic navigation is also increasing, particularly when tumor margins are obscure and bony anatomy is distorted or destroyed. ${ }^{28,34,35}$ These techniques improve real-time understanding of anatomy to improve surgical accuracy, protection of vital structures, and precision of cuts around tumor margins during the osteotomy. Considerations include radiation exposure, complication rates, and cost-effectiveness. Although these factors are understudied in oncological spine surgery, in degenerative disease, trauma, and deformity surgery they are largely equivocal, or even favorable, given low-dose CT and adequate surgical volume over which to distribute costs. $28,34,36-39$ This case contributes to the anecdotal benefit of navigation for rare, atypical, or particularly large spinal tumors. Such technology dovetails with $3 \mathrm{D}$ printing for complex spinal oncology surgeries, as both enable greater appreciation of relevant 3D surgical anatomy as it is explored.

Despite a successful operation from a technical standpoint and our best efforts, the patient experienced significant morbidity involving readmission, new neurological deficits, and likely recurrence of disease at 1 year. This highlights a necessary balance in decision-making for patients with complex spinal tumor: the risk of greater procedural morbidity from aggressive management versus that of increased recurrence after less aggressive management. En bloc resection of chordoma is well supported as one of the best options to reduce recurrence, ${ }^{2,3,7-10}$ and new technologies increase feasibility even for exceedingly complex tumors. ${ }^{13,27,28}$ However, in some cases, the longterm oncological outcome will be similar to that following a less morbid single-stage intralesional resection. Surgical morbidity must be weighed against the oncological benefits of aggressive resection, especially for such locally involved disease. We advocate that these complex cases are optimally managed at specialty centers where decisions can be made in the context of state-of-the-art technology and the availability of multidisciplinary teams.

\section{References}

1. McMaster ML, Goldstein AM, Bromley CM, et al. Chordoma: incidence and survival patterns in the United States, 1973-1995. Cancer Causes Control. 2001;12(1):1-11.

2. Mukherjee D, Chaichana KL, Adogwa O, et al. Association of extent of local tumor invasion and survival in patients with malignant primary osseous spinal neoplasms from the Surveillance, Epidemiology, and End Results (SEER) database. World Neurosurg. 2011;76(6):580-585

3. Mukherjee D, Chaichana KL, Gokaslan ZL, et al. Survival of patients with malignant primary osseous spinal neoplasms: results from the Surveillance, Epidemiology, and End Results (SEER) database from 1973 to 2003. J Neurosurg Spine. 2011;14(2): 143-150.

4. Smoll NR, Gautschi OP, Radovanovic I, et al. Incidence and relative survival of chordomas: the standardized mortality ratio and the impact of chordomas on a population. Cancer. 2013;119(11): 2029-2037.

5. Bakker SH, Jacobs WCH, Pondaag W, et al. Chordoma: a systematic review of the epidemiology and clinical prognostic factors predicting progression-free and overall survival. Eur Spine J. 2018;27(12):3043-3058.

6. Stacchiotti S, Casali PG, Lo Vullo S, et al. Chordoma of the mobile spine and sacrum: a retrospective analysis of a series of patients surgically treated at two referral centers. Ann Surg Oncol. 2010; 17(1):211-219.

7. Gokaslan ZL, Zadnik PL, Sciubba DM, et al. Mobile spine chordoma: results of 166 patients from the AOSpine Knowledge Forum Tumor database. J Neurosurg Spine. 2016;24(4):644-651.

8. Boriani S, Bandiera S, Biagini R, et al. Chordoma of the mobile spine: fifty years of experience. Spine (Phila Pa 1976). 2006;31(4): 493-503.

9. Barrenechea IJ, Perin NI, Triana A, et al. Surgical management of chordomas of the cervical spine. J Neurosurg Spine. 2007;6(5): 398-406.

10. D'Amore T, Boyce B, Mesfin A. Chordoma of the mobile spine and sacrum: clinical management and prognosis. J Spine Surg. 2018; 4(3):546-552.

11. Goomany A, Timothy J, Robson C, et al. En bloc resection of a thoracic chordoma is possible using minimally invasive anterior access: an 8-year follow-up. J Neurosci Rural Pract. 2016;7(1): 138-140. 
12. Liu S, Zhou X, Song A, et al. Surgical treatment of giant chordoma in the thoracic spine combining thoracoscopic and posterior spinal surgery: a case report. Medicine (Baltimore). 2019;98(35): e16990.

13. Luzzati AD, Shah S, Gagliano F, et al. Multilevel en bloc spondylectomy for tumors of the thoracic and lumbar spine is challenging but rewarding. Clin Orthop Relat Res. 2015; 473(3):858-867.

14. Sáenz AA, Sánchez MP. Management of a cervical chordoma involving C7-T1. Interdiscip Neurosurg. 2018;13:141-144.

15. Sciubba DM, Gokaslan ZL, Black JH 3rd, et al. 5-Level spondylectomy for en bloc resection of thoracic chordoma: case report. Neurosurgery. 2011;69(2 suppl operative):onsE248onsE256.

16. Shah AA, Paulino Pereira NR, Pedlow FX, et al. Modified en bloc spondylectomy for tumors of the thoracic and lumbar spine: surgical technique and outcomes. J Bone Joint Surg Am. 2017;99(17): 1476-1484.

17. Wang $X$, Eichbaum E, Jian F, et al. Two-stage en bloc resection of multilevel cervical chordomas with vertebral artery preservation: operative technique. Oper Neurosurg (Hagerstown). 2018; 14(5):538-545.

18. Zhang X-M, Fournel L, Lupo A, et al. En bloc resection of thoracic tumors invading the spine: a single-center experience. Ann Thorac Surg. 2019;108(1):227-234.

19. Bailey CS, Fisher CG, Boyd MC, etal. En bloc marginal excision of a multilevel cervical chordoma. Case report. J Neurosurg Spine. 2006;4(5):409-414.

20. Mavrogenis AF, Angelini A, Ruggieri P. Editorial about: modified en bloc spondylectomy for tumors of the thoracic and lumbar spine. J Spine Surg. 2019;5(2):296-299.

21. Missenard G, Bouthors C, Fadel E, et al. Surgical strategies for primary malignant tumors of the thoracic and lumbar spine. Orthop Traumatol Surg Res. 2020;106(1S):S53-S62.

22. Nazzaro JM, Arbit E, Burt M. "Trap door" exposure of the cervicothoracic junction. Technical note. J Neurosurg. 1994;80(2): 338-341.

23. Leary OP, Liu DD, Boyajian MK, et al. Complex wound closure by plastic surgery following resection of spinal neoplasms minimizes postoperative wound complications in high-risk patients. J Neurosurg Spine. 2020;33(1):77-86.

24. Ahmed AK, Pennington Z, Molina CA, et al. Multidisciplinary surgical planning for en bloc resection of malignant primary cervical spine tumors involving 3D-printed models and neoadjuvant therapies: report of 2 cases. J Neurosurg Spine. 2019;30(4):1-8.

25. Garofalo F, di Summa PG, Christoforidis D, et al. Multidisciplinary approach of lumbo-sacral chordoma: from oncological treatment to reconstructive surgery. J Surg Oncol. 2015;112(5):544-554.

26. Ahsan $F$, Inglis $T$, Allison $R$, et al. Cervical chordoma managed with multidisciplinary surgical approach. ANZ J Surg. 2011;81(5): 331-335.

27. Sciubba DM, Chi JH, Rhines LD, et al. Chordoma of the spinal column. Neurosurg Clin N Am. 2008;19(1):5-15.

28. Nasser R, Drazin D, Nakhla J, et al. Resection of spinal column tumors utilizing image-guided navigation: a multicenter analysis. Neurosurg Focus. 2016;41(2):E15.

29. Wilcox B, Mobbs RJ, Wu A-M, et al. Systematic review of 3D printing in spinal surgery: the current state of play. J Spine Surg. 2017;3(3):433-443.
30. Lador R, Regev G, Salame K, et al. Use of 3-dimensional printing technology in complex spine surgeries. World Neurosurg. 2020; 133:e327-e341.

31. Ozturk AM, Sirinturk S, Kucuk L, et al. Multidisciplinary assessment of planning and resection of complex bone tumor using patient-specific 3D model. Indian J Surg Oncol. 2019;10(1): 115-124.

32. Wang YT, Yang XJ, Yan B, et al. Clinical application of threedimensional printing in the personalized treatment of complex spinal disorders. Chin J Traumatol. 2016;19(1):31-34.

33. Xiao JR, Huang WD, Yang XH, et al. En bloc resection of primary malignant bone tumor in the cervical spine based on 3-dimensional printing technology. Orthop Surg. 2016;8(2):171-178.

34. Bandiera S, Ghermandi R, Gasbarrini A, et al. Navigationassisted surgery for tumors of the spine. Euro Spine J. 2013;22(6 suppl):S919-S924.

35. Rahmathulla G, Nottmeier EW, Pirris SM, et al. Intraoperative image-guided spinal navigation: technical pitfalls and their avoidance. Neurosurg Focus. 2014;36(3):E3.

36. O'Brien JR. The use of intraoperative CT and navigation for the treatment of spinal deformity in open and minimally invasive surgery. Spine (Phila Pa 1976). 2017;42(suppl 7):S28-S29.

37. Lee YC, Lee R. Image-guided pedicle screws using intraoperative cone-beam CT and navigation. A cost-effectiveness study. J Clin Neurosci. 2020;72:68-71.

38. Su AW, Luo TD, Mclntosh AL, et al. Switching to a pediatric dose 0 -arm protocol in spine surgery significantly reduced patient radiation exposure. J Pediatr Orthop. 2016;36(6):621-626.

39. Scarone P, Venier A, Porz N, et al. Reliability and importance of intraoperative CT navigation in spinal surgery: a single-center experience on a cohort of 235 cases. J Neurol Surg A Cent Eur Neurosurg. 2017;78(S 01):S1-S22.

\section{Disclosures}

Dr. Oyelese reports personal fees from DePuy-Synthes Spine and Brainlab for work outside the current study.

\section{Author Contributions}

Conception and design: Pertsch, Leary, Oyelese. Acquisition of data: Woo. Analysis and interpretation of data: Liu, Woo, Ng, Fridley. Drafting the article: Pertsch, Leary, Camara-Quintana, Liu. Critically revising the article: Pertsch, Leary, Camara-Quintana, Liu, Niu, Woo, Ng, Fridley, Gokaslan. Reviewed submitted version of manuscript: Pertsch, Leary, Liu, Niu, Woo, Ng, Oyelese, Fridley, Gokaslan. Approved the final version of the manuscript on behalf of all authors: Pertsch. Administrative/technical/material support: Woo, $\mathrm{Ng}$. Study supervision: $\mathrm{Ng}$, Oyelese.

\section{Supplemental Information}

Previous Presentations

This work was previously presented as an abstract at the Virtual 2020 International Chordoma Research Workshop, held July 16-17, 2020.

\section{Correspondence}

Nathan J. Pertsch: The Warren Alpert Medical School of Brown University, Providence, RI. nathan_pertsch@brown.edu. 